\title{
PROGRAMMING OF THE GENERALIZED NONLINEAR \\ PARAXIAL EQUATION FOR THE FORMATION \\ OF SOLITONS WITH MATHEMATICA
}

FREDERICK OSMAN AND ROBERT BEECH

Received 3 January 2002

We present the nonlinearity and dispersion effects involved in the propagation of optical solitons which can be understood by using a numerical routine to solve the generalized nonlinear paraxial equation. A sequence of code has been developed in Mathematica to explore in depth several features of the optical soliton's formation and propagation. These numerical routines were implemented through the use of Mathematica and the results give a very clear idea of this interesting and important practical phenomenon.

\section{Introduction}

The field of nonlinear optics has developed in recent years as nonlinear materials have become available and widespread applications have become apparent. This is particularly true for optical solitons and other types of nonlinear pulse transmission in optical fibres. Subsequently, this form of light propagation can be utilized in the future for very high capacity dispersion-free communications. The purpose of this paper is to describe the use of a very powerful tool to solve the generalized nonlinear paraxial equation that has stable solutions called optical solitons [1]. The solitary wave (or soliton) is a wave that consists of a single symmetrical hump that propagates at uniform velocity without changing its form. The physical origin of solitons is the Kerr effect, which relies on a nonlinear dielectric constant that can balance the group dispersion in the optical propagation medium. The resulting effect of this balance is the propagation of solitons, which has the form of a hyperbolic secant [6].

\section{Nonlinear paraxial equation}

The electric field is considered as a monochromatic wave propagating along the $z$-axis with the wave number $k$ and angular frequency $\omega$, that is, the field $E$ is assumed to be in the expansion form:

$$
E(r, \theta, z, t)=\sum_{l=-\infty}^{\infty} E_{l}(r, \theta, \xi, \tau ; \varepsilon) \exp [i(k z-\omega t)] .
$$


With $E_{-l}=E_{l}^{*}$ (complex conjugate) where $k_{l}=l k, \omega_{l}=l \omega$ and the summation is taken over all harmonics generated by the nonlinearity due to the Kerr effect and $E_{l}(r, \theta, \xi, \tau ; \varepsilon)$ is the envelope of the $l$ th harmonic changing slowly in $z$ and $t$. The slow variables $\xi$ and $\tau$ are defined by:

$$
\xi=\varepsilon^{2} z, \quad \tau=\varepsilon\left(t-\frac{z}{V_{g}}\right) .
$$

From (2.1) and (2.2), the displacement is found by:

$$
D=\varepsilon \times E=\sum D_{l} \exp \left[i\left(k_{l} z-\omega_{l} t\right)\right]
$$

It has been shown by [2] that $E_{l}(r, \theta, \xi, \tau ; \varepsilon)$ can be expanded in terms of $\varepsilon$ :

$$
E_{l}(r, \theta, \xi, \tau ; \varepsilon)=\sum_{n=1}^{\infty} \varepsilon^{n} E_{l}^{(n)}(r, \theta, \xi, \tau)
$$

From which the generalised nonlinear paraxial equation for $u_{1}^{(1)}(\xi, \tau)$ is obtained [4]:

$$
i \frac{\partial u}{\partial \xi}+\frac{1}{2} \frac{\partial^{2} u}{\partial \tau^{2}}+|u|^{2} u=-i \Gamma u+i \delta \frac{\partial^{3} u}{\partial \tau^{3}}
$$

The importance of (2.5) is that it can be solved into normalized reference coordinates. A clear view of the evolution of the envelope along the normalised propagation path results. This will also allow us to study the different cases, such as the classical situation, where $\Gamma=0$, which results in the standard nonlinear paraxial equation $[4,7]$.

2.1. Initial conditions. The solution of the nonlinear paraxial equation can be solved exactly by the inverse scattering method. A planar stationary light beam in a medium with a nonlinear refractive index can be described as a dimensionless form [7]:

$$
i \frac{\partial u}{\partial \xi}+\frac{\partial^{2} u}{\partial \tau^{2}}+k|u|^{2} u=0
$$

The method used to solve the exact inverse scattering method is applicable to equations of the type:

$$
\frac{\partial u}{\partial \xi}=\widehat{S}[u]
$$

where $\widehat{S}$ is a nonlinear operator differential in $z$, which can be represented in the form:

$$
\frac{\partial \widehat{L}}{\partial \xi}=i[\widehat{L}, \widehat{A}]
$$




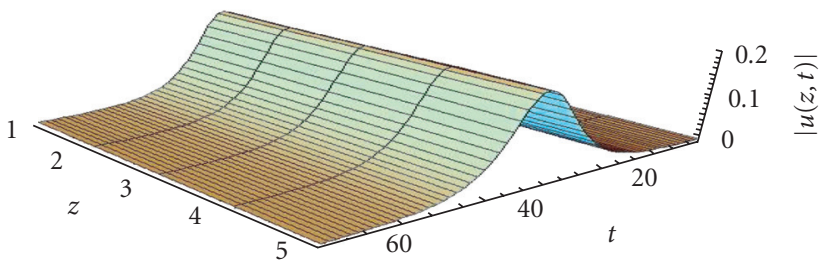

Figure 3.1. The simple soliton; pulse definition $2 \eta \operatorname{sech}\left[2 \eta\left(j-z_{0}\right)\right]$; displacement constant $z_{0}=32$, amplitude $\eta=0.1$, and time divisions, $\tau=64$.

Here $\widehat{L}$ and $\widehat{A}$ are linear differential operators containing the sought function $u(z, t)$ in the form of a coefficient. The result in (2.6) can be verified in (2.8) with the operator's $\widehat{L}$ and $\hat{A}$ taking the form of the nonlinear paraxial equation [4]:

$$
u(z, t)=2 \eta \operatorname{sech}\left[2 \eta\left(z-z_{0}\right)+8 \eta \xi t\right] \cdot \exp \left[i\left(-2 \xi x-4\left(\xi^{2}-\eta^{2}\right)\right) t+\phi\right],
$$

where $\eta, \xi, \phi, t, z_{0}$ are scaling parameters. This form of the solution can also be known as a soliton that has a stable formation. Using this solution and beginning at the origin $z=0$, a wave formation can be acknowledged by [4]:

$$
u(0, t)=\eta \operatorname{sech}\left[t-t_{0}\right]
$$

\section{Finite difference solution}

In order to compute a valid solution, $(2.10)$ is converted to a finite-difference equation $[4,5]$ using $z=\xi$ and $t=\tau$. The time discretisation will be indicated by an $n$ superscript and the spatial position will have an associated integer subscript $i$. Thus $u(z, t)$ is denoted by $u_{i}^{n}$. The various $z$ values become $i \Delta z$ where $\Delta z$ is the mesh width and $i=0,1,2, \ldots, I$. Similarly, the time variable becomes $n \Delta t$ where $\Delta t$ is the time step $n=0,1,2, \ldots, N$. Following a standard explicit procedure, the finite-difference version for (2.5) is found [4]:

$$
\begin{gathered}
i \frac{u_{i+1}^{n}-u_{i-1}^{n}}{2 \Delta z}+\frac{u_{i}^{n-1}-2 u_{i}^{n-1}+u_{i-1}^{n-1}}{2(\Delta t)^{2}}+\left|u_{i}^{n-1}\right|^{2} u_{i}^{n-1} \\
=-i \Gamma u_{i}^{n-1}+i \delta \frac{u_{i}^{n+2}-2 u_{i}^{n+1}+2 u_{i}^{n-1}-u_{i}^{n-2}}{2(\Delta t)^{3}} .
\end{gathered}
$$

The computer software Mathematica [2] has all the tools to code this finite-difference equation, to solve it and to produce the output graphics in two and three dimensions.

3.1. Numerical results. This section investigates a numerical result of the nonlinear paraxial equation (2.9), for the formation of solitons with Mathematica $[4,7]$ for the following cases: Figures 3.1 and 3.2 show the propagation of a simple soliton. Figure 3.3 shows two solitons plotted on the same graph. Figure 3.4 shows the propagation of a square wave soliton. Figure 3.5 shows propagation of a simple soliton with a phase angle. 


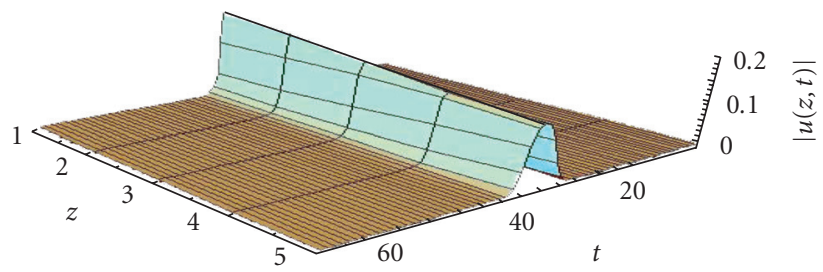

Figure 3.2. The effect of excluding the amplitude constant $\eta$ from the pulse definition. This graph is obtained from pulse $=2 \eta \operatorname{sech}\left[2 \eta\left(j-z_{0}\right)\right]$.

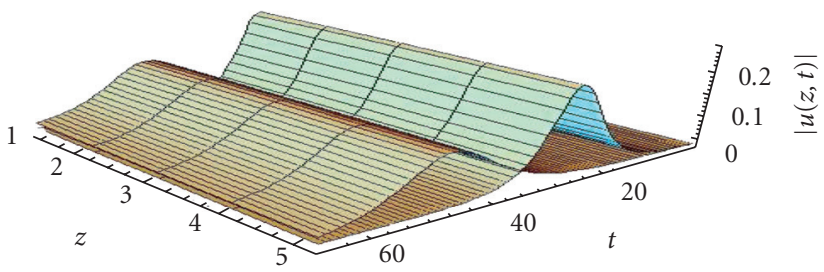

Figure 3.3. Two solitons with amplitude at $\eta=0.25$ and $\eta=0.1$ respective to $z_{0}=38$ and $z_{1}=32$; plotted on the same graph, to illustrate the difference made by varying the amplitude and lateral displacement.

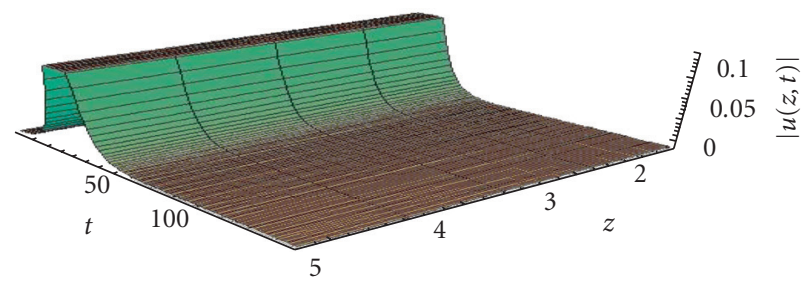

Figure 3.4. Square wave soliton.

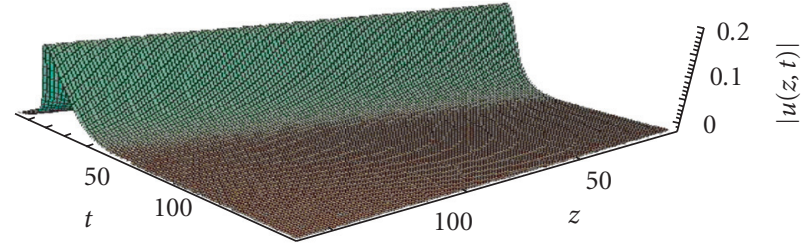

Figure 3.5. Here the program has been allowed to set the scales automatically, at $0-60$, to accommodate the square array in order that the program may successfully compile.

Figure 3.6 shows propagation of two solitons in phase. Figures 3.7-3.9 show propagation of two solitons in different stages of collisions. 


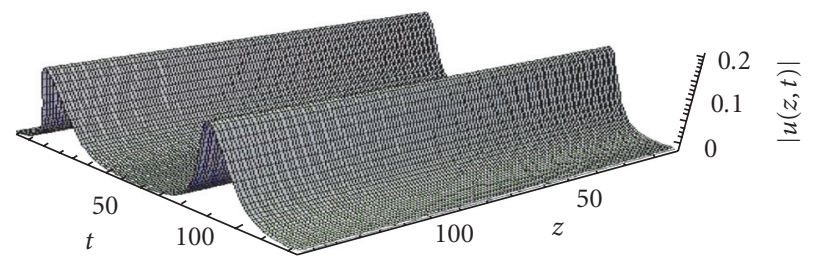

Figure 3.6. Two solitons following in phase.

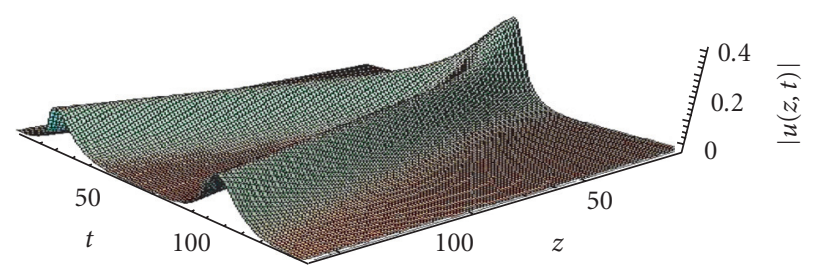

Figure 3.7. Two solitons in phase: the displacement constants are set at $z_{0}=30$ and $z_{0}=100$. Two solitons in early stage of collision.

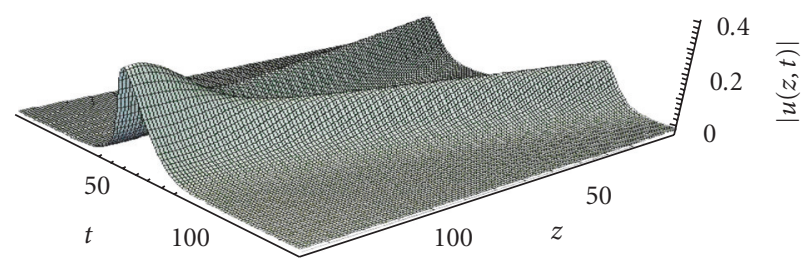

Figure 3.8. Two solitons in phase: the later displacement constants are set at $z_{0}=100$ and $z_{1}=30$.

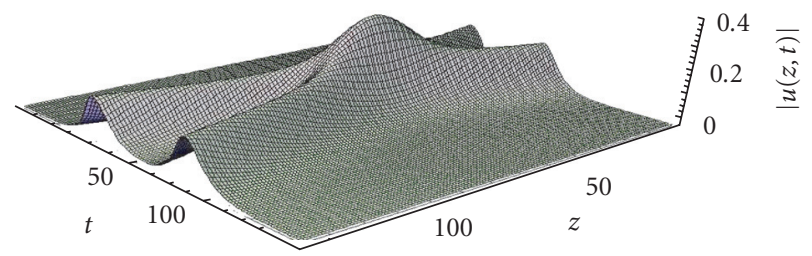

Figure 3.9. The final effect for the soliton collision with $z_{0}=80$ and $z_{1}=50$.

\section{Conclusion}

The formation and propagation of solitons has been observed and understood in this paper by implementing a numerical routine to solve the nonlinear paraxial equation [4, 7]. A sequence of code has been developed to explore in depth several features of the soliton's formation and propagation. This paper investigated changes in the solutions 
of the nonlinear paraxial equation when the parameters $\eta, \xi, \phi, t, z_{0}$ are modified in the following way. The parameter $\eta$ has most noticeable effect on the amplitude of the wave. When $\eta>0.1$, this condition usually encountered a square wave. When $\eta<0.1$, a normal plane soliton is resulted. Changing the inequality sign has no noticeable effect. The parameter $\xi$ gives the wave a phase angle. This phase angle becomes noticeable above 0.001. A phase angle above 0.1 tends to separate the soliton solution into a row of peaks. The results were found to be most useful at a phase angle of 0.07 . The direction of the phase changes with the sign. The parameter $t_{0}$ moves the soliton peak back and forth along the $t$-axis. Finally the timer divisions, on the same scale as $t_{0}$, are constant in the Mathematica program [4]. This constant investigates the spread of the graph along the $t$ axis. This constant was shown to be most useful when the time divisions were set at 64 . A time division of 128 was used in this paper at the stage soliton collisions [2]. Nonlinearity and dispersion effects involved in the propagation of optical solitons have been presented in graphical form, using a numerical routine for Mathematica.

\section{References}

[1] P. G. Drazin and R. S. Johnson, Solitons: An Introduction, Cambridge Texts in Applied Mathematics, Cambridge University Press, Cambridge, 1989.

[2] A. Hasegawa, Optical Solitons in Fibers, Springer, Berlin, 1989.

[3] H. A. Haus, Optical fiber solitons, their properties and uses, Proc. IEEE 81 (1993), no. 7, 970-983.

[4] F. Osman, Nonlinear paraxial equation at laser plasma interaction, Ph.D. thesis, University of Western Sydney, Sydney, 1998.

[5] G. D. Smith, Numerical Solution of Partial Differential Equations. Finite Difference Method, 3rd ed., Oxford Applied Mathematics and Computing Science Series, The Clarendon Press, Oxford University Press, New York, 1985.

[6] G. B. Whitham, Linear and Nonlinear Waves, Pure and Applied Mathematics, John Wiley \& Sons, New York, 1974.

[7] V. E. Zakharov and A. B. Shabat, Exact theory of two-dimensional self-focusing and onedimensional self-modulation of waves in nonlinear media, Soviet Physics JETP 34 (1972), no. 1, 62-69.

Frederick Osman: School of Quantitative Methods and Mathematical Sciences, University of Western Sydney, Penrith, NSW 1797, Australia

E-mail address: f.osman@uws.edu.au

Robert Beech: School of Quantitative Methods and Mathematical Sciences, University of Western Sydney, Penrith, NSW 1797, Australia

E-mail address: 98167845@day.uws.edu.au 


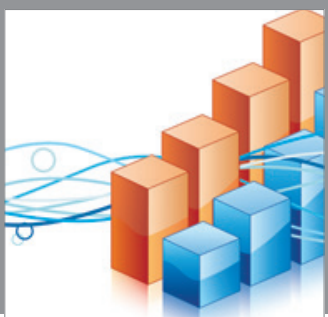

Advances in

Operations Research

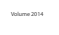

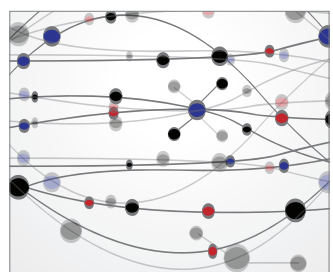

\section{The Scientific} World Journal
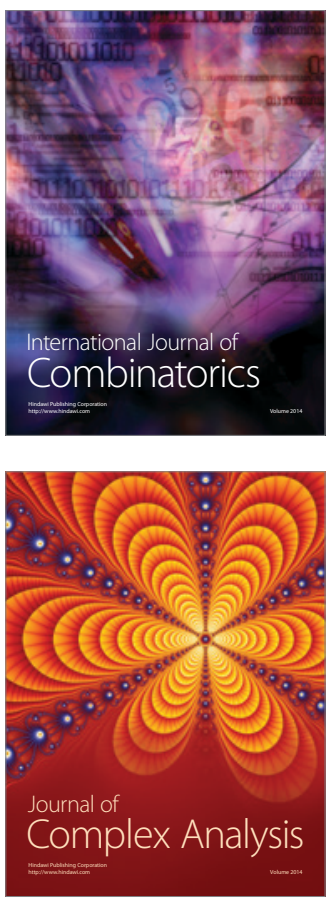

International Journal of

Mathematics and

Mathematical

Sciences
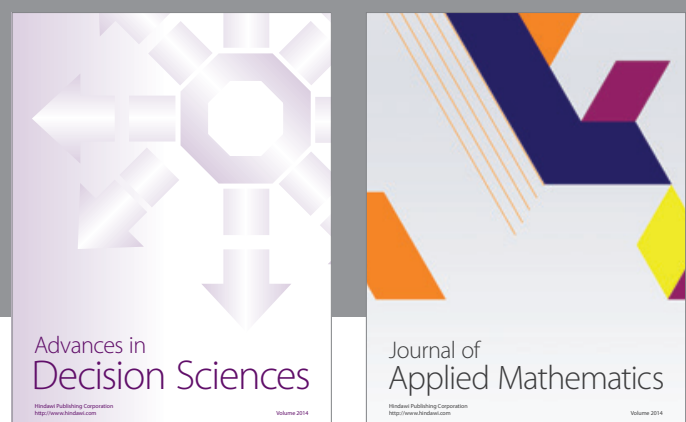

Journal of

Applied Mathematics
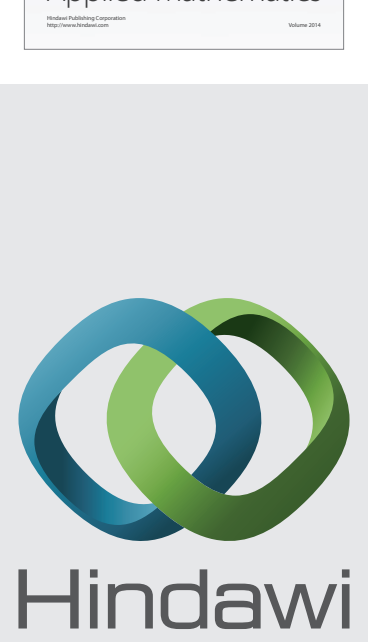

Submit your manuscripts at http://www.hindawi.com
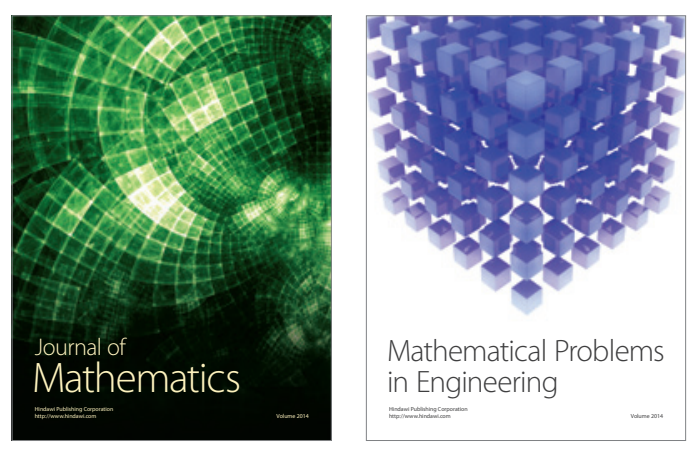

Mathematical Problems in Engineering
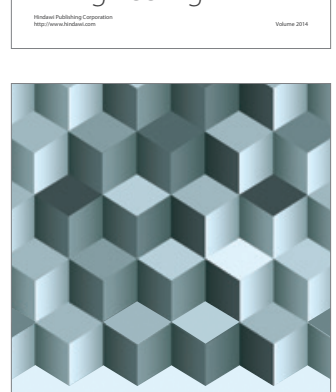

Journal of

Function Spaces
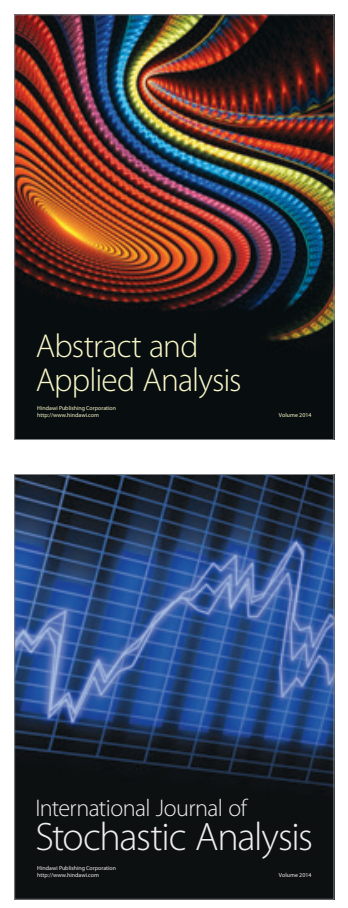

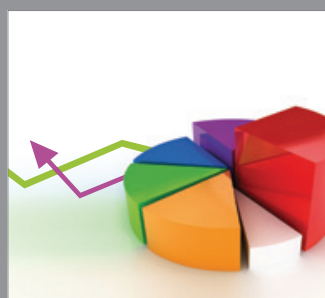

ournal of

Probability and Statistics

Promensencen
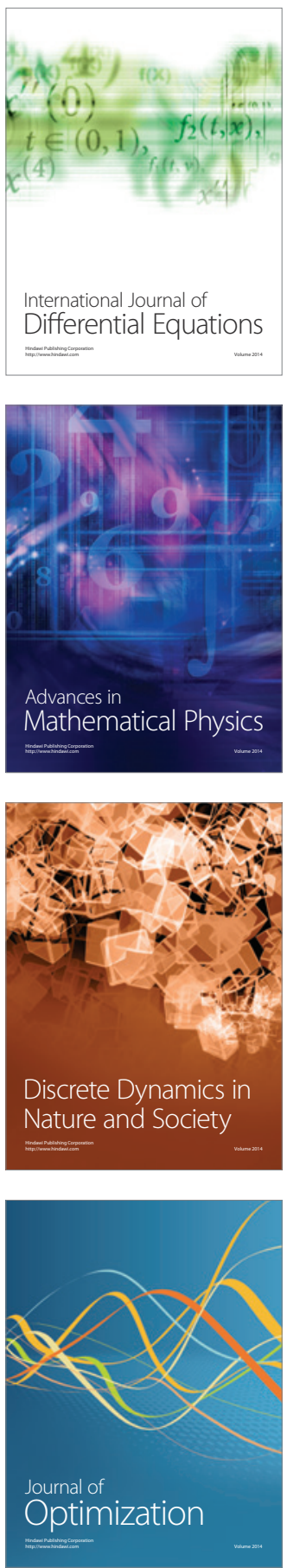